\title{
Advantages of A Novel Modulation technology Time Shifted Non-orthogonal Frequency Division Multiplexing
}

\author{
He Mingyan \\ Information Engineering College \\ Dalian University \\ Dalian, China \\ mingyan321@126.com
}

\begin{abstract}
Orthogonal frequency division multiplexing (OFDM) is a high efficient multi-carrier modulation technique for broadband communication applications with high peak to average power ratio (PAPR). In order to reduce the adverse effects of high PAPR on communication, a novel time shifted non-orthogonal frequency division multiplexing (TS-NFDM) technology is created by introducing the time-shift factor into multi-carrier modulation in this paper, which brings distinct advantages on bandwidth efficiency and PAPR. According to the analysis of constellation diagram and experiment results, the bandwidth efficiency of TS-NFDM is 4.29 times as higher as OFDM approximately. PAPR of TS-NFDM also could be reduced better than OFDM.
\end{abstract}

Keywords-OFDM; Time Shifted Non-orthogonal Frequency Division Multiplexing (TS-NFDM); bandwidth efficiency; PAPR

\section{INTRODUCTION}

Orthogonal frequency-division multiplexing ( OFDM ) has developed into a popular scheme for wideband digital communication, used in applications such as digital television, High Definition television, DSL Internet access, wireless networks, and 4G mobile communications. MIMI-OFDM has been the dominant air interface for $4 \mathrm{G}$ and $5 \mathrm{G}$ broadband wireless communications. The symbol rate of OFDM is the closest to the Nyquist rate[1]. There are some researches on breaking Nyquist rate without consideration of ISI and the change of minimum distance of signals in a signal space. Mazo showed that for ideal sinc pulses the symbol time can be reduced to $0.802 \mathrm{~T}$ without suffering any loss in minimum Euclidean distance [2], and the signaling rate can be increased with as much as $25 \%$. The subsequent researches [3], [4] have shown the methods of computing the minimum distance of faster-than-Nyquist (FTN) signaling. The time and frequency compressions turns out that spectral savings of at least $50 \%$ are possible in principle[5], the bandwidth per data symbol can be further reduced. A non-orthogonal modulation method has a much higher transmission rate than the traditional[6].

Time shifted non-orthogonal frequency division multiplexing (TS-NFDM) is established in this paper. The new modulation method which is improving [7], has much higher bandwidth efficiency than OFDM. Just like the character FDM of OFDM, TS-NFDM divides a radio channel into a large number of closely spaced sub-channels, but the sub-channels are non-orthogonal. TS-NFDM has the distinct advantages on bandwidth efficiency and peak to average power ratio (PAPR), which are brought by its time-shift character. The comparison of TS-NFDM and OFDM on bandwidth efficiency and PAPR is shown in this paper.

\section{THE WAVEFORM STRUCTURE OF TS-NFDM}

The signal of TS-NFDM can be described as following

$$
g(t)=\sum_{i=1}^{F} \sum_{j=1}^{Q_{i}} g_{i j}\left(t-\tau_{i j}\right)
$$

where $g_{i j}\left(t-\tau_{i j}\right) \quad$ is the sub-carrier $i$, $g_{i j}\left(t-\tau_{i j}\right)=\left\{\begin{array}{cc}\Phi_{i j}\left(t-\tau_{i j}\right) g o_{i j}\left(t-\tau_{i j}\right), & t \in T_{i j} \\ 0, & t \notin T_{i j}\end{array}\right.$ $\Phi_{i j}\left(t-\tau_{i j}\right)=\left\{\begin{array}{ll}a_{i j}, & t \in T_{i j} \\ 0, & t \notin T_{i j}\end{array}\right.$ is the amplitude of the sub-carrier $i j$, $T_{i j}$ denotes the valid period of the sub-carrier $i j$. The valid periods are mostly the same, but there are time-shifts between different valid periods. $g o_{i j}\left(t-\tau_{i j}\right)=\sin \frac{2 n \pi}{T_{0 i j}}\left(t-\tau_{i j}\right)$ is called the base sub-carrier, where $n=1,2 \cdots, 6, \cdots$. All of the sub-carriers are divided into $F$ groups. $T_{0 i j}$ is the period of base sub-carrier $j$ $\left(j=1, \cdots, Q_{i}\right)$ in group $i(i=1, \cdots, F)$, where $Q_{i}$ is the number of sub-carriers in group $i$. When $i=1, \cdots, F ; j=1, \cdots, Q_{i}$, there could be $Q_{i}=Q_{i+1}$ and $Q_{i} \neq Q_{i+1}, T_{0 i j}=T_{0 i(j+1)}$ and $T_{0 i j} \neq T_{0 i(j+1)}$. $U=\sum_{i=1}^{F} Q_{i}$ is the total number of base sub-carriers in one symbol. In other words, the frequencies of the base sub-carriers in one group are the same, the sub-carriers are included in the same sub-channel, and $F$ is the number of sub-channels. $\tau_{i j}$ denotes the time-shift of the sub-carrier $g_{i j}\left(t-\tau_{i j}\right) . T_{d}$ is the interval between two adjacent sub-carriers, which is the same 
value when $T_{d}=\frac{T_{11}}{U}$, where, $T_{11}$ is the valid period of the first sub-carrier in the symbol. An example of the waveform structure of TS-NFDM is illustrated in figure 1. Being similar to OFDM, the sub-carriers of TS-NFDM are divided into several sub-channels in the frequency field. The sub-channels of TS-NFDM are not be orthogonal in frequency field because of every sub-carrier of TS-NFDM has the different time-shift.

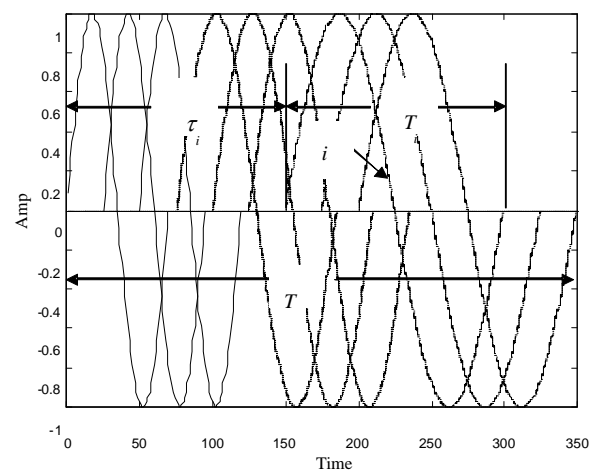

Fig.1. The structure of sub-carriers in TS-NFDM system

Demodulation of TS-NFDM is performed by solving a linear equation group as following: $\boldsymbol{A} \boldsymbol{x}=\boldsymbol{B}$, where $\boldsymbol{A}$ is the coefficient matrix of the equation group. It has the form as following: $\boldsymbol{A}=\left[a_{1 j} \cdots a_{i j} \cdots a_{U j}\right]^{\mathrm{T}}, j=1 \cdots U, \boldsymbol{x}=\left[x_{1}, \cdots, x_{U}\right]^{\mathrm{T}}$ is the sub-carriers' amplitude vector, $\boldsymbol{B}=\left[B_{1}, \cdots, B_{U}\right]^{\mathrm{T}}$.

The coefficient matrix $\boldsymbol{A}$ can be created by the series coherence operations as following:

$$
\begin{aligned}
\boldsymbol{B}_{i} & =\mathrm{O}_{\tau_{i}}^{T_{i}+\tau_{i}} \operatorname{gr}(t) \operatorname{gor}_{i j}\left(t-\tau_{i j}\right) d t \\
& =a_{i 1} x_{1}+\cdots+a_{i j} x_{j}+\cdots+a_{i U} x_{U}
\end{aligned} \quad(i, j=1, \cdots, U)
$$

where, $g r(t)=\sum_{i=1}^{F} \sum_{j=1}^{Q_{i}} g r_{i j}\left(t-\tau_{i j}\right)$ is the received signal, $g r_{i j}\left(t-\tau_{i j}\right)$ is the received sub-carrier, $g o r_{i j}\left(t-\tau_{i j}\right)$ is the received base sub-carrier.

The demodulation course can be depicted as:

$$
\boldsymbol{x}=\boldsymbol{A}^{-1} \boldsymbol{B}
$$

where $\boldsymbol{A}^{-1}$ is the inverse of the coefficient matrix $\boldsymbol{A}$ in the equation.

\section{OVERVIEW OF THE RESEARCH BASIS}

The comparison conditions are given as following: the subchannel bandwidth, power, noise, bit error rate and symbol period of TS-NFDM and OFDM are all the same.

According to the wave structure of TS-NFDM, there are different sub-channels included in one symbol. When the bandwidth efficiency ratio of one sub-channel between TSNFDM/S and QAM is calculated, the bandwidth efficiency ratio between TS-NFDM and OFDM will be given. Where, for simple expression, TS-NFDM/S denotes one sub-channel of TS-NFDM and QAM denotes one sub-channel of OFDM.

Thereinafter, we analyze the bandwidth efficiency of TSNFDM/S and QAM using the constellation diagram[8]. Defining the bandwidth efficiency ratio between TS-NFDM/S and QAM as following:

$$
\eta=\frac{R_{b N} / W_{N S}}{R_{b Q} / W_{Q}}
$$

where $W_{N S}$ and $W_{Q}$ denote the bandwidths of TS-NFDM/S and QAM respectively. The bit transmission rate can be expressed as following

$$
R_{b}=\frac{H}{T} \log _{2} M
$$

where, $M$ denotes number of the constellation points in eachdimension of constellation diagram and $H$ denotes number of the dimensions, number of the total constellation points can be defined as $L=M^{H}$ in the constellation diagrams [8].

An example of the waveform structures of TS-NFDM/S and QAM is illustrated in figure 2. Let $V_{N S}=\alpha V_{Q}, V_{N S}$ and $V_{Q}$ denote the sub-carrier amplitude of TS-NFDM/S and QAM respectively. Because the number of sub-carriers in TS$\mathrm{NFDM} / \mathrm{S}$ is larger than in QAM, there is $0<\alpha<1$. When the valid period of the sub-carrier of TS-NFDM/S equals to the period of the sub-carrier of QAM, there are $T_{N S}=2 T_{Q}$ and $W_{N S}=W_{Q}$, where $T_{N S}$ and $T_{Q}$ denote the symbol period of TSNFDM/S and QAM respectively. The bandwidth efficiency ratio is

$$
\eta=\frac{\log _{2} L_{N} / T_{N S}}{\log _{2} L_{Q} / T_{Q}}=\frac{H \log _{2}\left[\alpha\left(L_{Q}^{1 / 2}-1\right)+1\right]}{2 \log _{2} L_{Q}}
$$

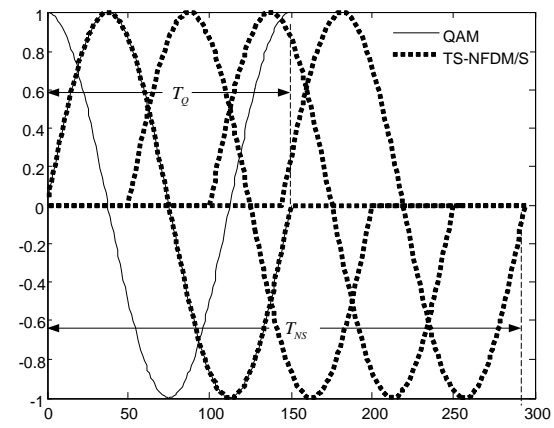

Fig. 2. The structure of TS-NFDM/S and QAM in one symbol (the subcarriers number of TS-NFDM/S could be more than four in practice)

Given that $B_{N S}=1 \mathrm{bit}, T_{N S}=1 /\left(2 \times 10^{3}\right) \mathrm{s}, V_{N S}=0.25$ volt and $P W_{s N}=P W_{s Q}$, it can be determined that the number of subcarriers of TS-NFDM/S is 33 and $\alpha=0.25, V_{Q}=1$ volt, $B_{Q}=2$ bit , where $B_{N S}$ denotes the number of bits of one sub- 
carrier of TS-NFDM/S, $B_{Q}$ denotes the number of bits of one sub-carrier of QAM, $P W_{s N}$ and $P W_{s Q}$ denote the powers of TSNFDM/S and QAM respectively. The bandwidth efficiency ratio $(\eta)$ is get to 4.125 .

When the number of the sub-channels of TS-NFDM and OFDM are determined as 10 for convenience of calculation without loss of generality when powers of TS-NFDM and OFDM are kept the same. The theoretical calculating value of bandwidth efficiency ratio between TS-NFDM and OFDM is as following: $\eta_{t h}=5.3625[8]$.

\section{THE EXPERIMENT OF COMPUTER SIMULATION AND PERFORMANCE ANALYSIS}

Based on the theoretical analysis of bandwidth efficiency ratio between TS-NFDM/S and QAM systems, we add noise to the modulated signal. Given one-sided PSD of white noise $65 \mathrm{dBm}$, the variables of TS-NFDM/S system are as follows: $H=33, B_{N S}=2$ bit, $V_{N S}=0.2$ volt, and the variables of QAM system are: $B_{Q}=4$ bit, $V_{Q}=0.5$ volt and $T_{Q}=1 /\left(4 \times 10^{3}\right) \mathrm{s}$. The center frequency of sub-carrier in TS-NFDM/S and QAM systems is $16 \times 10^{3} \mathrm{~Hz}$. The bit error probabilities of TSNFDM/S and QAM systems are 7.15e-4 and 6.61e-4 respectively.

Inserting the above experiment variables into (6), the bandwidth efficiency ratio between TS-NFDM/S and QAM systems is

$$
\eta_{s}=\frac{\log _{2} L_{N} / 2}{\log _{2} L_{Q}}=\frac{H \times B_{N S} / 2}{2 \times B_{Q}}=4.125
$$

Because of the bit error probabilities of TS-NFDM/S is bigger than QAM, the bandwidth efficiency ratio between TSNFDM/S and QAM should be less than this result.

The experiment of TS-NFDM and OFDM systems is similar to the experiment of TS-NFDM/S and QAM. Given one-sided PSD of white noise $-69 \mathrm{dBm}$, the variables of TSNFDM system are as follows: $H=33, B_{N}=2$ bit, $V_{N}=0.22$ volt, and the variables of OFDM system are: $B_{O}=5$ bit, $V_{O}=0.63$ volt, $T_{O}=1 /\left(4 \times 10^{3}\right) \mathrm{s}$. The sub-channel number of TS-NFDM and OFDM systems are both 10, the center frequencies of sub-carriers in each sub-channel are shown in table 1 . The bit errors of TS-NFDM and OFDM systems are 8.66e-4 and 5.47e-4 respectively.

Considering the using bandwidth of TS-NFDM system is 0.77 times of the using bandwidth of OFDM system, according to (4) and (5), the bandwidth efficiency ratio between TSNFDM and OFDM systems is

$$
\eta_{m}=\frac{\log _{2} L_{N} / W_{N} / 2}{\log _{2} L_{O} / W_{O}}
$$

Inserting the experiment value of variables into (7), the bandwidth efficiency ratio[between TS-NFDM and OFDM systems is

$$
\eta_{m}=\frac{H \times B_{N} / 2}{2 \times B_{O}} \times 1.3 \approx 4.29
$$

Because of the ill-posedness of the equation group increases along with raise of number of the sub-carriers in TSNFDM system, the experiment bandwidth efficiency ratio is different from the theoretical value[9]. For keeping the same bit error of TS-NFDM system, the bits of one sub-carrier of TS-NFDM should be decreased. The bit rate and bandwidth efficiency will be reduced.

TABLE I. THE CENTER FREQUENCIES OF SUB-CARRIERS IN EACH SUB-CHANNEL OF TS-NFDM AND OFDM SYSTEMS (UNIT: KHZ)

\begin{tabular}{|c|c|c|c|c|c|c|c|c|c|c|}
\hline & $f_{c}(1)$ & $f_{c}(2)$ & $f_{c}(3)$ & $f_{c}(4)$ & $f_{c}(5)$ & $f_{c}(6)$ & $f_{c}(7)$ & $f_{c}(8)$ & $f_{c}(9)$ & $f_{c}(10)$ \\
\hline OFDM & 16 & 20 & 24 & 28 & 32 & 36 & 40 & 44 & 48 & 52 \\
\hline TS-NFDM & 16 & 19 & 22 & 25 & 28 & 31 & 34 & 37 & 40 & 43 \\
\hline
\end{tabular}

\section{PAPR OF Ts-NFDM AND OFDM}

A large number of closely spaced orthogonal sub-carrier signals are used to carry data on parallel data streams or channels, the instantaneous power of the transmit signal probably would be much more than the average power. High peak-to-average-power ratio (PAPR) is one of the main limitations of OFDM systems, and high PAPR becomes the burden of hardware design.

Some efficient and distortion-less schemes for PAPR reduction, such as hard limiter, reducing the occurrence probability of the peak of the transmission signal, increasing the redundancy of the transmitted signal[10] and sub-carriers scheduling, can be adopted. TS-NFDM has other obvious advantages to reduce PAPR except the above methods. Firstly, due to the time-shift characteristic the increase of the maximum amplitude of TS-NFDM transmission signal, which is increased with the sub-carrier number, is lower than OFDM. In this case, TS-NFDM would have lower maximum amplitude of the transmission signal than that of OFDM. The relationship between the maximum amplitude of the transmission signal and the sub-carriers number of TS-NFDM and OFDM is shown in Figure 3. The different curves of TS-NFDM subcarriers with one cycle, two period and four period sine wave are given respectively; the increase of the maximum amplitude of the transmission signal with high frequency sub-carrier becomes lower when the number of sub-carriers becomes more.

The positive and negative amplitude of each sub-carrier in cross could cancel out the transmission signal amplitude because of the shift characteristics of TS-NFDM with respect to OFDM system. 


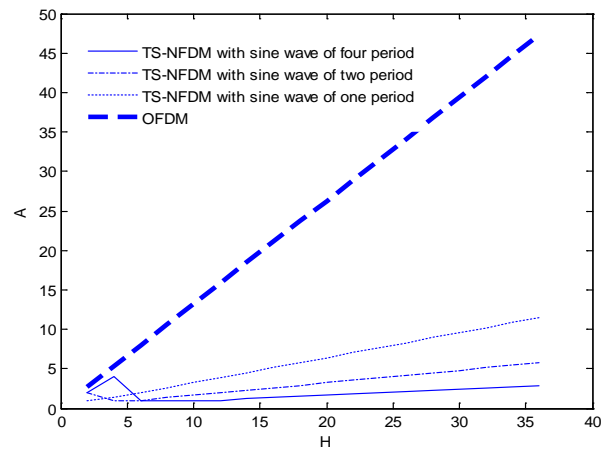

Fig. 3. The relationship between the maximum amplitude of the transmission signal and the sub-carriers number of TS-NFDM and OFDM

( $\mathrm{H}$ is the number of the sub-carriers, $\mathrm{A}$ is amplitude of the transmission signal. It should be noted that there are sudden jumps near the origin of TSNFDM curves with sub-carriers of two and four period, which is due to the time-shift of the TS-NFDM sub-carrier structure. In the case of small number of sub-carriers, the probability of the peak of each sub-carrier peak overlap results in the larger maximum amplitude of the transmission signal.)

Thus, we propose phase scrambling for the TS-NFDM system, which is not the same concept as traditional scrambling code, to reduce PAPR more effectively. The method of phase scrambling is that the positive phase (positive amplitude) is set as 1 , the negative phase (negative amplitude) is set as 0 . The 0,1 sequence is corresponding to the sub-carriers sequence. In this case, if there are continuous 0 or 1 in one symbol, it will lead to bigger peak. But we could process this binary sequence by scrambling, which could be called as randomization processing, to make it into a pseudo random sequence. A new sub-carriers group could be established according to the scrambled 0 and 1 sequence to form a low PAPR transmission signal.

Figure 4 shows the amplitudes of TS-NFDM transmission signal before and after the phase scrambling respectively. There are ten sub-carriers, each of which is four period sine wave with 15 sampling points in one period, when the maximum amplitude of the sub-carrier is 1 , PAPR of the transmission signal is 3.69 and 2.54 before and after the phase scrambling respectively. When using the experimental data in Table 1, PAPR of TS-NFDM is 5.15, and PAPR of OFDM is 6.2 after the phase scrambling.

\section{CONCLUSIONS}

Due to the time-shift characteristic, TS-NFDM system has some other unique characteristics. One of them could be thought of as many symbols of single-carrier modulation packed into one symbol, which is equivalent to increase the symbol rate greatly. Another is that the sub-channels of TSNFDM are not to be orthogonal, and there could be more subchannels than OFDM system in the same bandwidth. The bandwidth efficiency of TS-NFDM is higher than OFDM.

All of these intuitive analyses have been proved by the above-mentioned theories and experiments. The bandwidth efficiency of TS-NFDM is about 4.29 times of OFDM, and its PAPR is lower than OFDM.

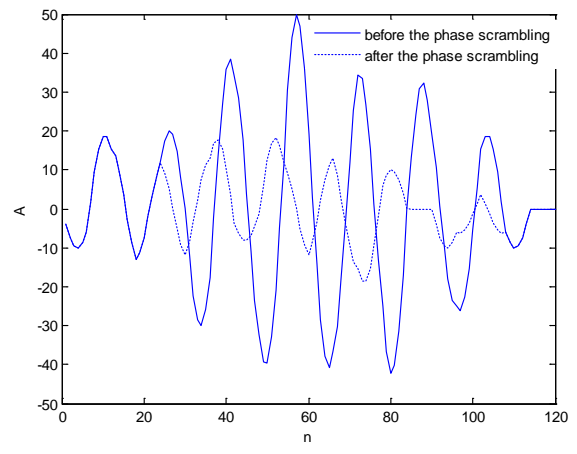

Fig. 4. The amplitudes of TS-NFDM transmission signal before and after the phase scrambling (where $\mathrm{n}$ is the sampling point, and $\mathrm{A}$ is the amplitude of transmission signal)

It should be noted that the ill-posedness of the demodulation equation group will reduce the decoding accuracy, which requires further researches. We have found a way to solve this problem, and the following research work is to further improve the performance of TS-NFDM.

\section{ACKNOWLEDGMENT}

This work was partly supported by the National Science Foundation of China under Grants 61401055.

\section{REFERENCES}

[1] Cao Qi-sheng. Research on Modulation Methods in Digital Communications Based on Non-orthogonal Ideas[D]. Dalian:Dalian Maritime University, 2009, pp. 28-31.(In Chinese)

[2] J E Mazo. "Faster-than-Nyquist signaling," Bell Syst Tech J, 1975, 54: pp.1451-1462.

[3] D Hajela. "On computing the minimum distance for faster-than-Nyquist signaling," IEEE Transactions on Information Theory, 1990, 36: pp. 289-295.

[4] J E Mazo, H J Landau. "On the minimum distance problem for fasterthan-Nyquist signaling," IEEE Transactions on Information Theory, 1988, IT-34: pp. 1420-1427.

[5] Fredrik Rusek, John B Anderson. "Multistream faster than Nyquist signaling," IEEE Transactions on Communications, 2009, 57(5): pp.1329-1340.

[6] Han Fang-ming. Wireless Digital Communications: A Timc-Frequency Perspective[D]. xi'an: xi'an Electronic and Science University, 2006, pp. 105-120. (In Chinese)

[7] Liang De-qun, Liang Wei-hua, Sun Chang-nian. "The phase-offset overlapped wave technique," Jounal of Electronics (China), 2003, 20(2): pp. 121-127.

[8] He Ming-yan, Liang De-qun, Cao Qi-sheng. "A Modulation with Higher Bandwidth Efficiency than OFDM," 2010 2nd International Conference on Signal Processing Systems. Dalian, 2010, pp. 393-397.

[9] Cao Qi-sheng, Liang De-qun. "Study on modulation techniques free of orthogonality restriction," Science in China, Series F, Information Sciences, 2007, 50(6), pp. 889-896.

[10] Jiang Xue-feng, Liang De-qun, Xing Yun-ting. "A non-linear mapping method for reducing the transmitted signals amplitude peak in multicarrier system," Journal of China Institute of Communications, 2001, 6: pp. 107-112. (In Chinese) 